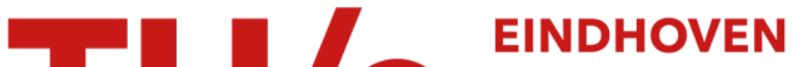 UNIVERSITY OF TECHNOLOGY
}

\section{Investigations of repetition rate stability of a mode-locked quantum dot semiconductor laser in an auxiliary optical fiber cavity}

Citation for published version (APA):

Breuer, S., Elsässer, W., Mclnerney, J. G., Yvind, K., Pozo, J., Bente, E. A. J. M., Yousefi, M., Villafranca, A., Vogiatzis, N., \& Rorison, J. M. (2010). Investigations of repetition rate stability of a mode-locked quantum dot semiconductor laser in an auxiliary optical fiber cavity. IEEE Journal of Quantum Electronics, 46(2), 150-157. https://doi.org/10.1109/JQE.2009.2033255

DOI:

10.1109/JQE.2009.2033255

Document status and date:

Published: 01/01/2010

Document Version:

Publisher's PDF, also known as Version of Record (includes final page, issue and volume numbers)

\section{Please check the document version of this publication:}

- A submitted manuscript is the version of the article upon submission and before peer-review. There can be important differences between the submitted version and the official published version of record. People interested in the research are advised to contact the author for the final version of the publication, or visit the DOI to the publisher's website.

- The final author version and the galley proof are versions of the publication after peer review.

- The final published version features the final layout of the paper including the volume, issue and page numbers.

Link to publication

\footnotetext{
General rights

- You may freely distribute the URL identifying the publication in the public portal. follow below link for the End User Agreement:

www.tue.nl/taverne

Take down policy

If you believe that this document breaches copyright please contact us at:

openaccess@tue.nl

providing details and we will investigate your claim.
}

Copyright and moral rights for the publications made accessible in the public portal are retained by the authors and/or other copyright owners and it is a condition of accessing publications that users recognise and abide by the legal requirements associated with these rights.

- Users may download and print one copy of any publication from the public portal for the purpose of private study or research.

- You may not further distribute the material or use it for any profit-making activity or commercial gain

If the publication is distributed under the terms of Article 25fa of the Dutch Copyright Act, indicated by the "Taverne" license above, please 


\title{
Investigations of Repetition Rate Stability of a Mode-Locked Quantum Dot Semiconductor Laser in an Auxiliary Optical Fiber Cavity
}

\author{
Stefan Breuer, Member, IEEE, Wolfgang Elsäßer, Senior Member, IEEE, John G. McInerney, Kresten Yvind, \\ Jose Pozo, Erwin A. J. M. Bente, Member, IEEE, Mirvais Yousefi, Member, IEEE, Asier Villafranca, \\ Nikos Vogiatzis, and Judy Rorison, Member, IEEE
}

\begin{abstract}
We have investigated experimentally the pulse train (mode beating) stability of a monolithic mode-locked multi-section quantum-dot laser with an added passive auxiliary optical fiber cavity. Addition of the weakly coupled $(\approx-24 \mathrm{~dB})$ cavity reduces the current-induced shift $d \nu / d I$ of the principal peak in the RF spectrum (the effective pulse repetition frequency) by more than an order of magnitude, from -39.5 to $-2.3 \mathrm{kHz} / \mathrm{mA}$. The $\mathrm{rms}$ timing jitter of the pulse train is simultaneously reduced from 1.4 to 0.9 ps.
\end{abstract}

Index Terms-Laser absorbers, laser resonators, laser stability, semiconductor lasers.

\section{INTRODUCTION}

I NVESTIGATION of the pulse trains emitted by mode-locked semiconductor lasers with a view to improving their stability and precision is crucial for the viability of application of these lasers in optical sampling, clocking, ranging, tomography, clock recovery and data stream regeneration, optical storage, two-photon fluorescence and microscopy [1], [2] among other applications. In particular, control and stabilization of the pulse repetition rate and reduction of inter-pulse timing jitter are essential. Many diverse frequency stabilization

Manuscript received June 15, 2009; revised August 04, 2009. Current version published December 18, 2009. This work was supported by the COST 288 research action "Nanoscale and Ultra fast Photonics."

S. Breuer and W. Elsäßer are with the Institute of Applied Physics, Technische Universität Darmstadt, 64289 Darmstadt, Germany (e-mail: stefan.breuer@physik.tu-darmstadt.de; elsaesser@physik.tu-darmstadt.de).

J. G. McInerney is with the Physics Department/Tyndall Institute, Lee Maltings, National University of Ireland, University College, Cork, Ireland (e-mail: J.McInerney@ucc.ie).

K. Yvind is with the DTU Fotonik Department of Photonics Engineering, Technical University of Denmark, Kgs. Lyngby DK-2800, Denmark (e-mail: ky@com.dtu.dk).

J. Pozo and E. A. J. M. Bente are with the Department of Electrical Engineering, COBRA Institute, Opto-Electronic Devices Group, Technische University of Eindhoven, NL-5612 AZ Eindhoven, The Netherlands (e-mail: J.M. Pozo.Torres@tue.nl; E.A.J.M.Bente@tue.nl).

M. Yousefi is with TNO-Science and Industry, 2600AD Delft, The Netherlands (e-mail: mirvais.yousefi@tno.nl).

N. Vogiatzis and J. Rorison are with the Centre for Communications Research, Department of Electrical and Electronic Engineering, University of Bristol, BS8 1TR, U.K. (e-mail: nik.vogiatzis@bristol.ac.uk; judy.rorison@ bristol.ac.uk).

A. Villafranca is with the Instituto de Investigación en Ingeniería de Aragón (I3A), Laboratorios Avanzados de Investigación Universidad de Zaragoza, 22197 Cuarte (Huesca), Spain (e-mail: asiervv@unizar.es).

Color versions of one or more of the figures in this paper are available online at http://ieeexplore.ieee.org.

Digital Object Identifier 10.1109/JQE.2009.2033255 techniques have been studied intensively: optical feedback, external cavity feedback, electronic locking or electronic servo control. Electronic stabilization of the pulse repetition rate and reduction of timing jitter by a factor of $\sim 7.5(500 \mathrm{~Hz}-100 \mathrm{MHz})$ have been demonstrated [3]. Other techniques include hybrid stabilization using optical feedback from a fiber-optical cavity resulting in a beat frequency linewidth reduction by a factor of $10^{3}$ [4]. Reduction of timing jitter of a ring dye laser by a factor of $2.2(5 \mathrm{kHz}-50 \mathrm{kHz})$ by referencing the cavity frequency to an external resonator has been reported [5]. Timing jitter reduction of a mode-locked semiconductor laser by referencing to an external atomic clock by a factor of $10(1 \mathrm{~Hz}-100 \mathrm{MHz})$ has been reported [6]. Another possibility of enhancing pulse train stability is the application of non-frequency selective external feedback. The stabilization potential of a combination of a passively mode-locked semiconductor laser with an optical fiber cavity and non-frequency selective mirror has yielded picosecond pulses with high peak power under continuous current injection [1], [7], [8]. An antireflective coated InGaAsP-InP $1.3 \mu \mathrm{m}$ two-section laser with proton-bombarded trench section in a passive external mirror cavity resulted in passive mode-locking at harmonics of the cavity round-trip time [9]. Weak resonant optical feedback has been employed to stabilize the fundamental beat frequency of a GaAlAs laser in a confocal reference cavity [4]. By applying optical feedback to a two-section semiconductor laser in conjunction with hybrid mode-locking the laser at a fixed modulation frequency on the short section, a considerable reduction of the timing jitter of a factor of 3.4 was reported [10]. Weak fiber-optical feedback leads to significant reduction in the central RF peak of close to two orders of magnitude when applied to a multi quantum well three-section laser [11]. Besides, the rate of change of the RF peak (effective pulse repetition rate) with current gives an indication on the stabilization influence. For a $\mathrm{CW}$ single-section laser diode with an external resonator, frequency pulling $d \nu / d I$ of $400 \mathrm{kHz} / \mathrm{mA}$ of the RF peak was reported [12]. For multi quantum well semiconductor ring lasers a frequency pulling $d \nu / d I$ and $d \nu / d T$ of $-64 \mathrm{kHz} / \mathrm{mA}$ and $-2.5 \mathrm{MHz} / \mathrm{K}$ are observed [2]. Much recent research on mode-locked quantum dot lasers has focused on comprehensive investigations on the pulse-to-pulse timing jitter performance and minimization of rms jitter [13]-[15].

In this paper we experimentally study the potential of a weakly coupled passive auxiliary optical cavity to stabilize 
the central beat frequency (effective pulse repetition rate) of a mode-locked quantum dot laser. The empty auxiliary cavity is a fixed-length configuration and is expected to reduce the deleterious effects of inevitable carrier fluctuations through injection current $I$ and reverse-bias voltage $U_{\mathrm{RB}}$ variations which induce drifts of the central RF peak $\nu_{0}$ as well as unintentional amplitude and frequency noise. By applying optical feedback to a mode-locked semiconductor laser via a passive auxiliary cavity, we expect constructive feedback at distinct frequencies of the auxiliary cavity modes that are spaced by the inverse of the delay of the auxiliary cavity, i.e., the formation of a mode comb. As the optical delay in the auxiliary cavity is constant, referencing and stabilizing the principal peak of the beat frequency spectrum to the inverse of the round-trip time is anticipated. Optimum pulse train stabilization is expected by matching the circulating pulses propagating in the main laser cavity with those in the auxiliary cavity. Given that quantum dot lasers can exhibit reduced dispersion and increased gain relative to quantum well lasers, the former should make ideal candidates for studying effects of optical feedback on stabilization of the RF beat spectra [16].

The paper is structured as follows: in the following section we describe the feedback configuration we applied to our modelocked quantum dot laser. We then present the results of experimentally observed radio-frequency spectra in free-running operation as well as with weak optical feedback intended to stabilize the central beat frequency. We then investigate the effects of different feedback conditions on the pulse timing jitter and quantify the reduction of timing jitter under appropriate feedback. Subsequently we discuss the obtained results by describing the stabilizing influence of the auxiliary cavity on the timing jitter distribution. Finally, potential to reduce externally stimulated strong timing jitter is demonstrated.

\section{EXPERIMENTAL RESULTS AND DISCUSSION}

The device under investigation is a commercially available fiber coupled multi-section quantum dot based semiconductor laser from Innolume $\mathrm{GmbH}$ (Former Nanosemiconductor $\mathrm{GmbH})$. The active medium consists of a Dot-in-a-well (DWELL) structure of 10 layers of InGaAs quantum dots embedded into GaAs. As schematically indicated in the inset of Fig. 1, the laser chip is processed into $3 \mu \mathrm{m} \times 300 \mu \mathrm{m}$ long absorber sections, each separated by a $700 \mu \mathrm{m}$ long gain section. Next to these sections, six long and three short sections are operated as gain sections such that a total laser cavity length of $\approx 8 \mathrm{~mm}$ is formed. The device is operated at a constant heat sink temperature of $20^{\circ} \mathrm{C}$. The gain sections are driven by a precision DC current source and a reverse bias from a DC voltage source is applied to the absorbing sections. All measurements were performed at a fixed reverse-bias voltage of $U_{\mathrm{RB}}=-5 \mathrm{~V}$, verified to be well within the mode-locking regime of the laser module.

Stability of the fundamental beat frequency was investigated experimentally, with precise control of the applied amount of weak optical feedback by a passive fiber optical auxiliary cavity. A total accessible feedback range of -24.2 to $-73.2 \mathrm{~dB}$ estimated at the laser facet is obtained. The experiment is



Fig. 1. Experimental configuration for investigating the influence of delayed optical feedback of an all fiber-based passive auxiliary cavity on the RF spectral stability of a quantum dot mode-locked laser. The external feedback is controlled by a variable reflector. Optionally, a set of optical fiber lengths, providing multiple incoherent or distributed feedback, allows investigation of strong external feedback on the beating stability and the timing jitter. The inset schematically depicts the electrical contacting scheme for gain and absorber sections.

shown schematically in Fig. 1. Feedback is provided by directly connecting the fiber-coupled output of the laser module to a fiber-coupled variable optical reflector. The resulting actual fiber length of the feedback setup is $5.3 \mathrm{~m}$, resulting in a mode comb separated by $\Delta \nu \approx 19.9 \mathrm{MHz}$. The variable optical back-reflector provides continuous tuning of the feedback fraction $\chi$ at the laser facet, with effective reflection coefficient varying between -73.2 to $-24.2 \mathrm{~dB}$. A $-3 \mathrm{~dB}$ coupling efficiency between the fiber and the laser is reasonably assumed and has to be taken into account additionally. A fiber-coupled InGaAs p-i-n photo-detector (Newport 400FC) was used to monitor the amount of optical feedback. A fiber-coupled high-speed InGaAs photo-detector ( $\mathrm{U}^{2} \mathrm{~T}$ XPDV 10220R; bandwidth $50 \mathrm{GHz}$ ) was connected to a broadband electrical spectrum analyzer (Agilent E4448A PSA series; bandwidth $50 \mathrm{GHz}$ ) to analyze the emission dynamics of the laser under optical feedback in the radio-frequency domain. A pair of single-stage optical isolators, each with an isolation of $>25 \mathrm{~dB}$ at $1.3 \mu \mathrm{m}$, ensure that back-reflections from the photo-detector were suppressed. Polarization effects of the fiber components were excluded by maximizing the radio-frequency amplitude response and a stable mounting of the optical components on the experimental bench.

\section{A. Fundamental Laser Characterization}

Prior to investigating the RF peak (mode beating) stability of the laser with fiber optical feedback, we measured output powers and SHG auto-correlation pulse widths. Fig. 2 shows the optical output power $P(I)$, the reverse-bias current $I_{\mathrm{SA}}$ in the absorbing sections, the auto-correlation pulse widths $\tau$ and amplitude $A_{\tau}$ of the optical auto-correlation signal for increasing injection and fixed reverse-bias $U_{\mathrm{RB}}=-5 \mathrm{~V}$. At that reverse 


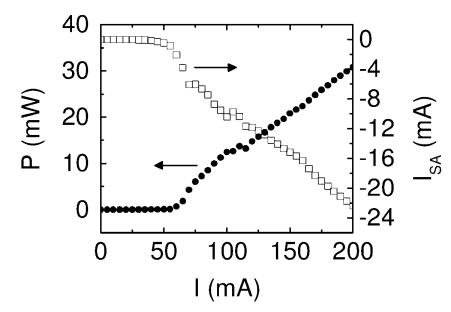

(a)

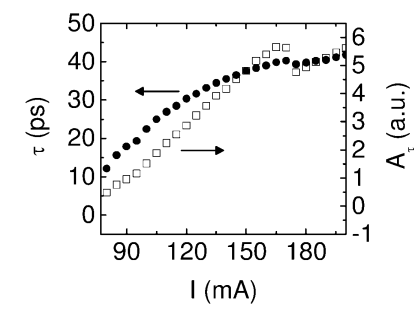

(b)
Fig. 2. Measured laser output power $P(I)$ and saturable absorber reverse-bias current $I_{\mathrm{SA}}$ (a), deconvolved SHG auto-correlation function width $\tau$ and autocorrelation signal amplitude $A_{\tau}$ (b) as functions of the injection current $I$. The laser is operated in mode-locked condition without optical feedback. A constant reverse-bias voltage of $U_{\mathrm{RB}}=-5 \mathrm{~V}$ was used to bias the saturable absorber sections.

bias, the laser threshold current is $60 \mathrm{~mA}$ (threshold current density $J_{0}$ is $211 \mathrm{~A} / \mathrm{cm}^{2}, 21.1 \mathrm{~A} / \mathrm{cm}^{2}$ per DWELL layer). Average output power is $12.5 \mathrm{~mW}$ at $96 \mathrm{~mA}$. Pulses are emitted above $80 \mathrm{~mA}$ with minimum deconvolved width of $12.1 \mathrm{ps}$ FWHM. The auto-correlation pulse peak power evolves linearly with increasing injection current. At $168 \mathrm{~mA}$ a step-like decrease in auto-correlation amplitude and a simultaneous slight discontinuity in the pulsewidth is caused by an artifact which stems from manually adjusting the gain setting of the auto-correlator. We identified proper mode-locked operation by simultaneously observing a sharp increase in intensity of the RF peak accompanied by a small RF linewidth $<50 \mathrm{kHz}$ as well as a sharp reduction of timing jitter. The auto-correlation function contrast in the applied non-collinear second harmonic generation (SHG) background-free intensity auto-correlator was measurement limited so we were not able to measure the background. Additionally, no low frequency noise was observed within the investigated operating regime. Detailed measurements of the SHG auto-correlation pulse width, amplitude and shape with the auxiliary cavity were compared to passively mode-locked condition without feedback. No shortening or significant broadening of the optical pulses and no change in the pulse shape due to the linear, passive auxiliary cavity were observed. For that reason, detailed auto-correlation studies under low feedback were not performed. The width of the longitudinal multimode spectrum $(-10 \mathrm{~dB})$ increases with increasing injection current from $5.0 \mathrm{~nm}$ at $96 \mathrm{~mA}$ to $6.0 \mathrm{~nm}$ at $132 \mathrm{~mA}$. The maximum spectral width is $8.7 \mathrm{~nm}$ at $198 \mathrm{~mA}$. The central emission wavelength at $200 \mathrm{~mA}$ was $1245 \mathrm{~nm}$. Together with the increase of the pulsewidth [Fig. 2(b)] this indicates that the mode-locking covers not all available longitudinal modes.

\section{B. Central Beat Frequency Stability}

Experimental studies of the stability of the mode-locked RF spectrum and subsequent timing jitter analysis were performed at the fundamental beat frequency using single-sideband phase noise measurement procedures. We concentrate on the analysis of the mode-locking beat frequency and the radio-frequency spectral width and amplitude, respectively. The corresponding beat spectra were recorded as a function of the total current to the gain sections. All measurements were performed at a fixed reverse bias voltage of $-5 \mathrm{~V}$. For all investigations we focus on injection currents below $120 \mathrm{~mA}$, as experimentally derived

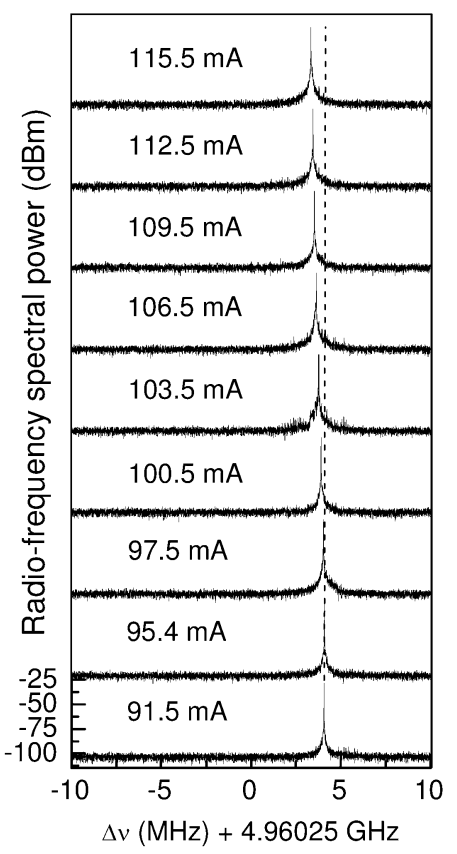

Fig. 3. Evolution of the RF spectra of the mode-locked pulse trains without feedback (solitary or free-running operation) at a fixed reverse bias voltage $U_{\mathrm{RB}}=-5 \mathrm{~V}$ and varying injection current. Spectrum analyzer settings: $5 \mathrm{kHz}$ resolution bandwidth, $1 \mathrm{kHz}$ video bandwidth filter. The scaling shown for the lower curve is valid for all traces. However, the traces are vertically offset for better visibility. The dashed line indicates the frequency $\nu_{0}$ at $91.5 \mathrm{~mA}$ as a guide for the eye.

maps of mode-locking suggest the optimum mode-locked regime lies below that injection current. The evolution of the radio-frequency power spectrum of the laser in passively mode-locked operation is shown in Fig. 3. We observed stable mode-locking with picosecond optical pulses starting at $91.5 \mathrm{~mA}$ where the fundamental central beat frequency of $\nu_{0}=4.9643 \mathrm{GHz}$ is observed. Proper mode-locking is indicated, amongst others, by a strong signal-to-noise ratio of $>60$ $\mathrm{dB}$. While increasing the injection current up to $115.5 \mathrm{~mA}$, the central beat frequency gradually shifts from $\nu_{0}$ towards shorter frequencies. A total frequency pulling $d \nu_{0} / d I$ of $-39 \mathrm{kHz}$ is observed up to $115.5 \mathrm{~mA}$. We then applied optical feedback $\chi=-53.6 \mathrm{~dB}$ and recorded more RF spectra. Fig. 4(a) illustrates the evolution of the power spectral density of the RF beat spectra for this feedback level.

We observed a two-fold effect while increasing the injection current. Firstly, the central RF peak $\nu_{0}$ appears in conjunction with a set of equally-spaced narrow peaks forming modulation sidebands. We will refer to this set of narrow lines as the mode comb of the auxiliary cavity. However, the observed mode-comb spacing does not amount to $19.9 \mathrm{MHz}$ but to $6.5 \mathrm{MHz}$, i.e., one third, corresponding to an optical path length of $8.6 \mathrm{~m}$ (group index of the fiber 1.45). We attribute this sub-harmonic effect to a longer time scale amplitude or phase modulation of the train of pulses inside the external cavity. At an injection current of $91.8 \mathrm{~mA}$, the central beat frequency is $\nu_{0}=4.9651 \mathrm{GHz}$. By increasing the current to $94.2 \mathrm{~mA}$, a switch of $\Delta \nu$ towards higher frequencies to $\Delta \nu^{\prime}=\Delta \nu_{0}-\Delta \nu=4.9714 \mathrm{GHz}$ is observed. Between 106.2 and $109.2 \mathrm{~mA}$, a switch back to $\Delta \nu$ appears, followed 


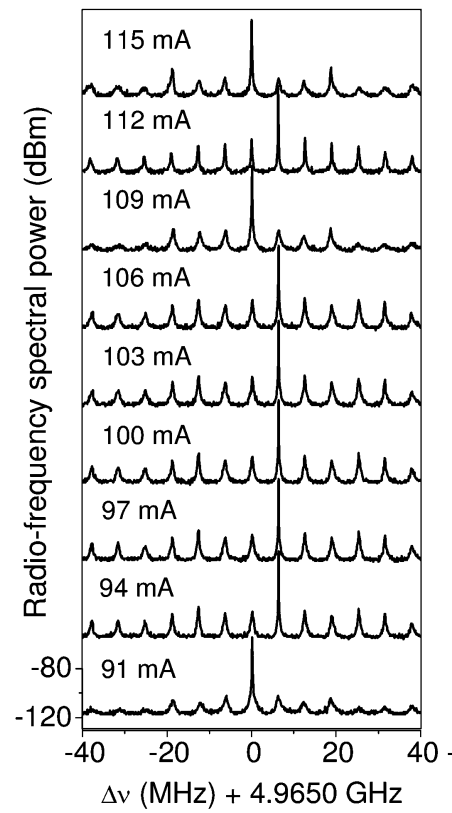

(a)

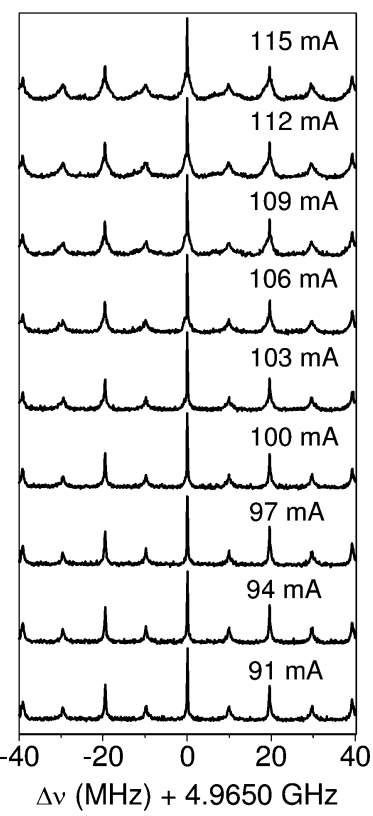

(b)
Fig. 4. Evolution of RF power spectra of the mode-locked pulse trains with feedback of $\chi=-53.6 \mathrm{~dB}$ (a) and $-24.2 \mathrm{~dB}$ (b) at a fixed reverse bias voltage $U_{\mathrm{RB}}=-5 \mathrm{~V}$ and varying injection current. Multiple switches of the beating carrier on the auxiliary comb modes are observed (a). Increased stability of the RF spectra is indicated by minimal shift of the central peak (b). Spectrum analyzer settings: $0.5 \mathrm{kHz}$ resolution bandwidth, $0 \mathrm{~Hz}$ video bandwidth filter. The scaling shown for the lower curve is valid for all traces. However, the traces are vertically offset for better visibility.

by an additional switch between $109.2 \mathrm{~mA}$ and $112.8 \mathrm{~mA}$ to $\Delta \nu^{\prime}$ and back to $\Delta \nu \approx 4.965 \mathrm{GHz}$ at $115.2 \mathrm{~mA}$. We attribute this asymmetric detuning to a non-vanishing alpha-factor [17]. We now increase the feedback $\chi$ to $-24.2 \mathrm{~dB}$ and compare the RF beat spectra in Fig. 4(b) with Fig. 4(a). There are two trends which can be identified. At $91 \mathrm{~mA}$ the central peak is $4.9651 \mathrm{GHz}$ and the dominant mode-comb frequency spacing is expected $\Delta \nu=19.5 \mathrm{MHz}$. Additionally, the next higher harmonic side-mode frequency equal to $0.5 \times \Delta \nu$ evolves. The relative position of the central beat frequency changes with reduced slope $d \nu_{0} / d I$ of $-4 \mathrm{kHz} / \mathrm{mA}$ within the regime from $91.2 \mathrm{~mA}$ to $115.2 \mathrm{~mA}$. Hence, the main RF peak is conspicuously robust against variations of the injection current over the complete range of investigated injection currents.

We observed clear stabilization of the mode-locking central beat frequency-the effective pulse repetition rate-by applying an empty, passive, auxiliary cavity to the laser. The reduced slope clearly indicates that interaction between the laser cavity and auxiliary cavity is established so that the mode-locking central beat frequency $\nu_{0}$ adapts itself or locks to the modes of the auxiliary cavity mode comb. At that point, the main cavity beat frequency is an integer multiple of the round-trip frequency (nominal mode spacing) of the auxiliary cavity.

\section{Phase-Noise-, Timing Jitter Stability and the Existence of a Feedback Threshold}

Complementary to the investigations on the pulse repetition frequency stabilization and the associated auxiliary cavity modes, we investigated the single-sideband phase noise and rms timing jitter $\sigma_{\text {rms }}$ in solitary operation and under weak optical feedback. An estimate of the root-mean-square timing jitter $\sigma_{\text {rms }}$ can be obtained by integration of the single-sideband noise spectral density $L(\nu)$ of phase fluctuations per $\mathrm{Hz}$ of the central beat frequency $\nu_{0}$ between specified low and the high frequency limits $\nu_{\text {low }}$ and $\nu_{\text {high }}$ [18], [19]

$$
\sigma_{\mathrm{rms}}=\left(1 / 2 \pi \nu_{0}\right) \sqrt{2 \int_{\nu_{\text {low }}}^{\nu_{\text {high }}} L(\nu) d \nu}
$$

The method of direct detection by radio-frequency analysis allows estimation of the total rms timing jitter, consisting of both amplitude and phase noise contributions. We investigate timing jitter in the free-running laser and then as function of feedback and injection current. We determine jitter in a frequency interval from $\nu_{\text {low }}=20 \mathrm{kHz}$ to an upper offset-frequency limit $\nu_{\text {high }}$ chosen to be half of the side-mode spacing frequency $\nu_{\text {high }}=\Delta \nu / 2$. This upper integration frequency, which was applied in the solitary as well as the feedback case, was chosen for two reasons. First, we expect the majority of the jitter to arise in close proximity to the central peak [18]. Second, we suppose that the modes created from the external cavity are caused in first approximation by pure amplitude modulation. Under this assumption, all apparent auxiliary cavity modes contain the same fundamental jitter. This assumption is based on the jitter contributions of increasing auxiliary cavity modes, starting at the fundamental beat frequency (mode number $k=0$ ) up to the maximum detectable cavity mode at $k=17$ and $115.8 \mathrm{~mA}$. We experimentally observe that the integrated timing jitter marginally changes with increasing number of auxiliary cavity mode with $d \sigma_{\mathrm{rms}} / d k=-0.001 \mathrm{ps}$. This corresponds to a relative change of $-0.36 \%$. By studying the timing jitter up to $\Delta \nu / 2$, we thus avoid to integrate multiples of the fundamental jitter.

In the following we investigate the evolution of the phase noise spectra within an integration range from $20 \mathrm{kHz}$ to $3.25 \mathrm{MHz}$ to be consistent with $\nu_{h i g h}=\Delta \nu / 2$. Fig. $5 \mathrm{de}-$ picts the obtained phase noise spectra for injection currents of $98.4 \mathrm{~mA}$ (a) and $115.2 \mathrm{~mA}$ (b). At optical feedback levels $\chi=-73.2 \mathrm{~dB}$ and $-43.8 \mathrm{~dB}$, the noise spectral density evolves at $-20 \mathrm{~dB} /$ decade. At the maximum feedback of $\chi=-24.2$ $\mathrm{dB}$, a steeper slope of $\sim-15 \mathrm{~dB} /$ decade is measured. By increasing the feedback level from $-73.2 \mathrm{~dB}$ to $-24.2 \mathrm{~dB}$, distinct reduction of the phase noise is observed. A maximum reduction of $14 \mathrm{~dB}$ is observed close to the peak at an lower offset frequency of $20 \mathrm{kHz}$. At $98.4 \mathrm{~mA}$, the reduction decreases to $3 \mathrm{~dB}$ at an offset frequency of $300 \mathrm{kHz}$. The corresponding timing jitter dependence on the injection current at the feedback levels of $\chi=-73.2 \mathrm{~dB},-43.8 \mathrm{~dB}$ and $-24.2 \mathrm{~dB}$ is depicted in Fig. 6(a). At minimum feedback of $-73.2 \mathrm{~dB}$, the timing jitter evolution exhibits large variations and a clear trend is not evident. By increasing the feedback to $\chi=-43.8 \mathrm{~dB}$ no major changes in the spectra are observed, besides a tendency to lower timing jitter $\approx 2$ ps at injection currents above 105 $\mathrm{mA}$. However, at a maximum feedback level of $-24.2 \mathrm{~dB}$, we observe a two-fold effect. On the one hand, we measure the lowest timing jitter in the presence of feedback: $(0.87 \pm 0.1)$ ps $(20 \mathrm{kHz}-3.25 \mathrm{MHz})$ at $98.4 \mathrm{~mA}$. On the other hand, by 


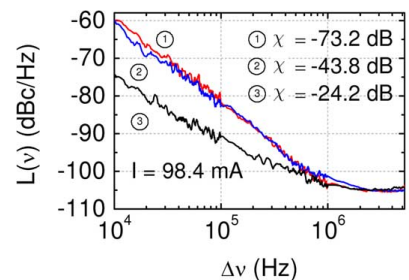

(a)

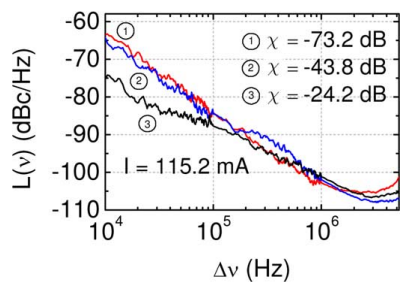

(b)
Fig. 5. Analysis of the single-sideband phase-noise spectra evolution as a function of the offset frequency range of $20 \mathrm{kHz}$ to $3.25 \mathrm{MHz}$. Phase-noise spectra with varying optical feedback $\chi$ from $-73.2 \mathrm{~dB}$ to $-24.2 \mathrm{~dB}$ at a fixed injection current of $98.4 \mathrm{~mA}$ (a) and $115.2 \mathrm{~mA}$ (b) and at a fixed reverse-bias voltage of $U_{\mathrm{RB}}=-5 \mathrm{~V}$.

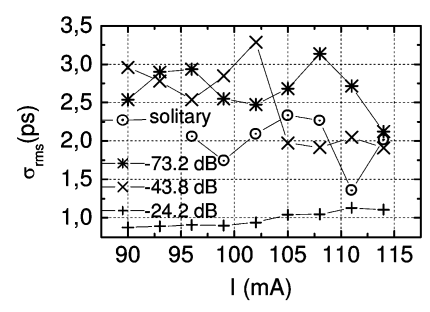

(a)

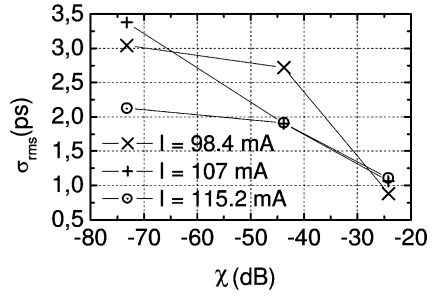

(b)
Fig. 6. Timing jitter $\sigma_{\text {rms }}$ extracted from the single-sideband phase noise spectra in Fig. 5. Evolution of the timing jitter within a frequency interval from $20 \mathrm{kHz}$ to $3.25 \mathrm{MHz}$ as a function of the injection current and applied feedback $\chi$ as well as in free-running or solitary operation (a). Timing jitter evolution for three injection currents as a function of applied feedback $\chi$ (b).

increasing the injection current from $98.4 \mathrm{~mA}$ to $115.2 \mathrm{~mA}$, monotonic increase of the timing jitter to $(1.1 \pm 0.1)$ ps is found. By investigating the influence of the feedback level on the timing jitter in Fig. 6(b), a substantial lowering of the timing jitter is observed for increasing feedback levels. This is indicated by a reduction of the timing jitter of a factor of 3.5 at 98.4 $\mathrm{mA}, 3.2$ at $106.8 \mathrm{~mA}$ and 1.9 at $115.2 \mathrm{~mA}$. By comparing the free-running timing jitter to that with feedback, an overall reduction of the timing jitter is clearly observed. The maximum reduction amounts to a factor of 2.2 at an injection current of $105 \mathrm{~mA}$.

This improvement of the timing jitter can be related to the previously observed interaction between the pulses propagating inside the active region of the laser and inside the auxiliary cavity, as schematically sketched in Fig. 7, in the following way. The three graphs in the left column of Fig. 7(a)-(c) schematically depict relative pulse positions, referenced to an ideal mode comb of equidistant comb spacing, the three graphs in the right column represent the associated timing jitter distributions. Firstly, we assume that the lengths of the main cavity and the auxiliary cavity is an integer multiple of the number of pulses inside the main laser cavity for most effective feedback. We then postulate that there is an ideal beat frequency $\nu_{0}$, where mode-locking is optimum. Secondly, we suppose that a pulse has a random time deviation from the ideal pulse position referenced to the ideal comb with a period proportional to the inverse beat frequency, i.e., the jitter and that this deviation has a distribution of width $w$ [Fig. 7(a)]. Additionally, we assume that an internal pulse accrues some random time deviation relative to a pulse returning from the auxiliary cavity. Both pulses should have the same distribution of this time deviation as they all arise from the same carrier population variations. Furthermore, we suppose an interaction mechanism between pulses inside the laser cavity, e.g., based on mutual cross-saturation of gain or loss, or refractive index coupling, or any other fast mechanism involving the nonlinear semiconductor medium [Fig. 7(b)]. The latter assumption is supported by experimentally observed locking or snapping of the central beat frequency to a mode of the auxiliary cavity. With these assumptions it is reasonable to assume that by coupling an auxiliary cavity to the laser an internal pulse of amplitude $A_{i}(\Delta t)$ and an external pulse $A_{e}(\Delta t)$ propagate inside the main cavity, resulting in two independent time deviation distributions $p_{i}(\Delta t)$ and $p_{e}(\Delta t)$, and that a nonlinear interaction of both internal and external pulse leads to a smaller time deviation distribution $p_{n}(\Delta t)$ of width $w_{n}<w_{e, i}$. [Fig. 7(c)]. The result of this interaction of pulses can be modeled by calculating the width $w\left\langle\Delta t_{n}\right\rangle$ of the resulting time deviation distribution of two pulses following $w\left\langle\Delta t_{n}\right\rangle>w\left\langle\left(\Delta t_{i}+\Delta t_{e}\right) / 2\right\rangle$ with $\Delta t_{i, e}$ being random time deviations for internal pulse $A_{i}$, and external pulse $A_{e}$ whose elements are uniformly distributed in an arbitrarily chosen interval $(-1,1)$. Hence, the new distribution of the average time deviation has a smaller width $w_{n}$ than the distribution $w$ of a single pulse [Fig. 7(c)]. This can be understood as follows. In most cases both internal and external pulses have an opposite time deviation which then combine to yield a smaller time deviation. At an assumed feedback of $100 \%$ and an interaction strength of $100 \%$ a reduction of the width $w_{n}$ of the timing jitter distribution of $\approx 30 \%$ is expected by applying a passive cavity for auxiliary mode comb enhancement. This consideration is initially valid for one round-trip, thus multiple-roundtrips may additionally help in reducing the timing jitter because after each round-trip the width of the distribution iteratively decreases.

We have observed a pronounced improvement of the stability of the central beat frequency and, in conjunction, a reduction of the timing jitter under weak optical feedback. Moreover, we also see the existence of a feedback threshold or interaction threshold by studying timing jitter with increasing injection current and varying feedback in Fig. 6(a). Starting at a feedback of $\chi=-43.8 \mathrm{~dB}$ and $105 \mathrm{~mA}$, a steplike decrease of the timing jitter is measured, whereas at a feedback of $-73.2 \mathrm{~dB}$ no such effect is observed. By increasing the feedback to $-24.2 \mathrm{~dB}$ a conspicuously strong reduction in the timing jitter occurs, as already discussed. This sudden stabilization at the highest applied feedback level suggests that a threshold exists i.e., minimum feedback level where the central RF peak is stabilized strongly. In the following we will refer to that phenomenological threshold as a feedback threshold [20]. This peculiarity is possibly related to an interaction between pulses in the main laser cavity and the auxiliary cavity as outlined above. Configuring the external feedback in a way that this feedback threshold is exceeded, stabilization of the central RF peak together with reduction of the timing jitter is expected. However, below that threshold, the system is less stable as evident by increased timing jitter and a RF peak not locked to a single auxiliary cavity mode. 


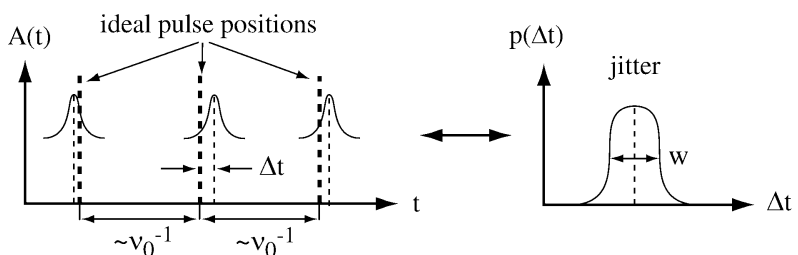

(a)

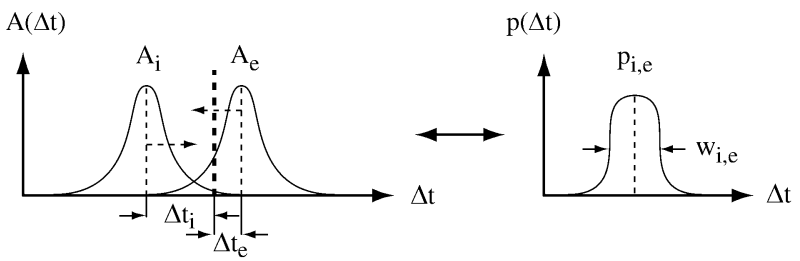

(b)

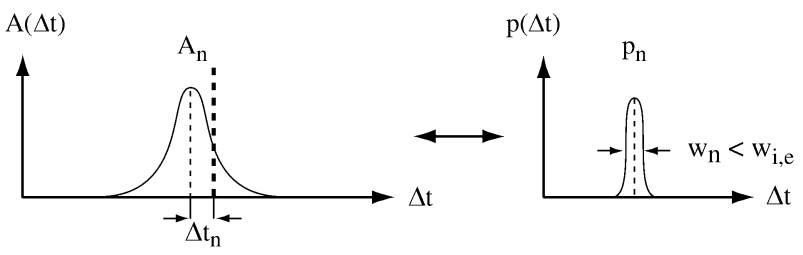

(c)

Fig. 7. Schematic of the possible interaction between internal and external pulses in the length matched active laser cavity and the auxiliary cavity leading to a reduction of timing jitter. (a) Pulses with amplitude $A(t)$, originating from the internal cavity, deviate from the dashed bold equidistant lines representing the ideal pulse positions for pulses without jitter. Resulting distribution of time deviation $p(\Delta t)$, the jitter, of the pulses. (b) By coupling the auxiliary cavity to the laser an internal pulse of amplitude $A_{i}(\Delta t)$ and an external pulse $A_{e}(\Delta t)$ propagate inside the active cavity, both leading to two independent time deviation distributions $p_{i}(\Delta t)$ and $p_{e}(\Delta t)$, respectively. Dashed arrows indicate pulse interaction direction. (c) Nonlinear interaction of internal and external pulse in (b) results in a smaller time deviation distribution $p_{n}(\Delta t)$ of width $w_{n}<w_{e, i}$. (a) Time domain representation of timing jitter, (b) Prior to auxiliary cavity assisted nonlinear pulse-pulse interaction, (c) Result of auxiliary cavity assisted nonlinear pulse-pulse interaction.

\section{Potential of Reducing Strong, Externally Stimulated Timing Jitter}

Data in the previous section showed that internal jitter is reduced by an auxiliary cavity. We now test that hypothesis further by adding more timing jitter artificially to see if this can also be reduced. In addition to its theoretical interest, the question of how strong external jitter can be reduced is significant given that real fiber-optic systems exhibit various sources of fluctuations, such as temperature or mechanical stress. In order to generate strong external jitter it is not sufficient merely to increase the feedback level as a stabilizing effect will occur, as observed in the timing jitter analysis. For that reason, a large amount of weak jitter is essential in order to ensure sub-feedback threshold condition. Also the sources of this jitter need to be asynchronous to avoid coherent superposition effects of discrete jitter components.

To satisfy these strict requirements, a slight modification in the setup in Fig. 1 is needed: introducing a variety of optical fiber patch cables of different lengths, interconnected via commercially available fiber adapters, in front of the variable reflector, should generate broad-spectrum feedback. This together with additional feedback from a $80 \mathrm{~m}$ long single mode optical fiber, ensuring that also low frequency components add to the distributed feedback, should cause strong increase in the timing jitter, to test if this can be suppressed in our system. At first we again investigated the stability of the central RF peak, followed then by the analysis of the timing jitter under various levels of optical feedback $\chi$. We applied optical feedback $\chi=-30.5 \mathrm{~dB}$ to the laser and recorded again the radio-frequency spectra as a function of the injection current. The resulting beat spectra are shown in Fig. 8. In contrast to the spectra for solitary operation in Fig. 3, the most pronounced property of the spectra under applied feedback $\chi$ is the presence of a broad noise pedestal over the complete investigated injection current range. The width of the pedestal is $>20 \mathrm{MHz}$. This noise pedestal is generated by the distributed feedback and appreciably broadens the fluctuation spectra. The central beat frequency is $\nu_{0}=4.9634 \mathrm{GHz}$ at $93.8 \mathrm{~mA}$ as shown in the lowest trace in Fig. 8 and indicating an initial frequency pulling of $0.9 \mathrm{MHz}$. By increasing the injection current from $93.8 \mathrm{~mA}$ to $103.4 \mathrm{~mA}$, the relative position of the central RF peak changes with a slope $d \nu_{0} / d I$ of $-43 \mathrm{kHz} / \mathrm{mA}$ to $\nu_{0}=4.9629 \mathrm{GHz}$. On further increasing the injection current up to $115.4 \mathrm{~mA}$ a substantially reduced slope of -2.33 $\mathrm{kHz} / \mathrm{mA}$ (from 103.4 to $115.4 \mathrm{~mA}$ ) is observed. As reported for the case of weak optical feedback, the corresponding spectra are depicted in Fig. 4(a) and (b), we explore here an equivalent improvement of the beating stability of the mode-locking carrier frequency $\nu_{0}$. We subsequently investigated the evolution of the phase noise spectra and related timing jitter under combined master (long $80 \mathrm{~m}$ fiber) and distributed feedback condition. The corresponding integrated timing jitter $\sigma_{\text {rms }}$ obtained at two laser injection currents is plotted in Fig. 9. At an injection current of $98 \mathrm{~mA}$ we observe an increase of the timing jitter with increasing feedback level. However at $115 \mathrm{~mA}$ we observe strongly increased timing jitter of $7.5 \mathrm{ps}(20 \mathrm{kHz}-0.5 \mathrm{MHz})$ at the lowest feedback setting of $-46.5 \mathrm{~dB}$, as estimated by the combined master and distributed feedback. The increased feedback leads to reduction of timing jitter to $2 \mathrm{ps}(20 \mathrm{kHz}-0.5 \mathrm{MHz})$ at the maximum applied feedback of $\chi=-30.5 \mathrm{~dB}$.

Consistent with the investigated timing jitter evolution under weak optical feedback, we see here both strong enhancement of timing jitter, as intended, and also reduction of the externally stimulated jitter, by increasing the feedback strength up to $-30.5 \mathrm{~dB}$. One additional effect is noteworthy in Fig. 8. Above a certain feedback threshold, in this case above an injection current of $101 \mathrm{~mA}$, modes of the auxiliary mode comb with a constant spacing of $\Delta \nu$ of $\approx 1 \mathrm{MHz}$ evolve. Simultaneously the central beat frequency is stabilized as indicated by a minimized shift with increasing injection current. In addition, the timing jitter evolution at an injection current of $98 \mathrm{~mA}$ supports our findings in that the jitter increases with increasing feedback below a threshold. Above that threshold, the feedback provided by the auxiliary cavity starts to become effective and, as already expressed, the existence of such a feedback threshold is consistently observed.

\section{CONCLUSION AND OUTLOOK}

We have performed systematic measurements of the mode beating stability of a $\approx 5 \mathrm{GHz}$ monolithic mode-locked multisection quantum dot laser emitting at $\approx 1250 \mathrm{~nm}$ by detailed 


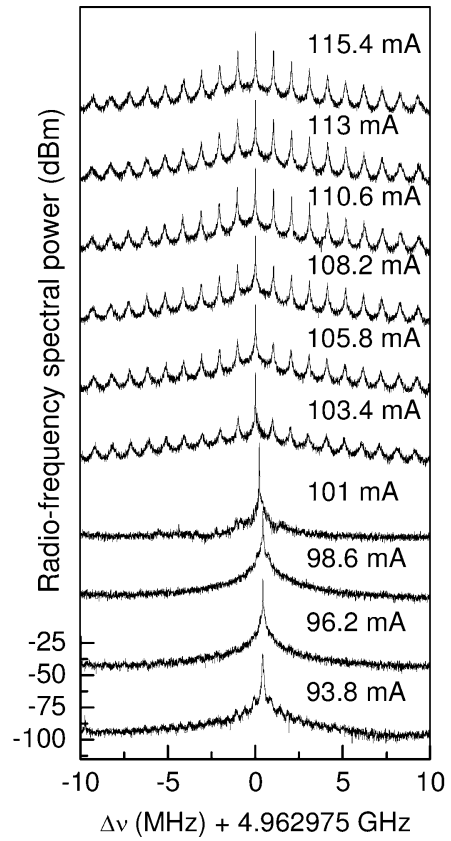

Fig. 8. Evolution of the radio-frequency power spectra of the mode-locked pulse trains obtained with distributed feedback applied to generate artificially strong timing jitter. The spectra are depicted for different injection currents at feedback $\chi=-30.5 \mathrm{~dB}$. A reverse bias voltage of $U_{\mathrm{RB}}=-5 \mathrm{~V}$ is applied. The scaling shown for the lower curve is valid for all traces. However, the traces are vertically offset for better visibility. Spectrum analyzer settings: $10 \mathrm{kHz}$ resolution bandwidth and $1 \mathrm{kHz}$ video bandwidth filter.

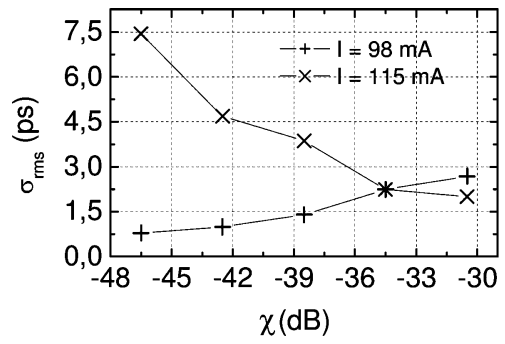

Fig. 9. Timing jitter $\sigma_{\text {rms }}$ extracted from the single-sideband phase noise spectra (Fig. 8) as functions of the feedback $\chi$ and for two injection currents of $97.8 \mathrm{~mA}$ and $115.2 \mathrm{~mA}$ and applied combined master and distributed feedback. The integration range is $20 \mathrm{kHz}$ to $0.5 \mathrm{MHz}$.

experimental studies of the radio-frequency spectra and particularly its central peak $\nu_{0}$ which dominates the pulse repetition frequency. By applying fiber-based passive optical feedback from an auxiliary cavity to attain an equally spaced auxiliary mode comb, we avoid any mechanical and temperature dependences of optically coupling the laser light. We elucidate the fact that delayed optical feedback from a passive auxiliary cavity has a two-fold effect depending on the operating parameters of the laser as well as the feedback level. By choosing injection current settings for optimum mode-locking and accurate control of the feedback, a ten-fold improvement of the central beat frequency stability over the whole injection current range is achieved, compared to the current tuning of the solitary laser. Hence, we demonstrate the potential of a simple passive, weakly coupled auxiliary cavity to substantially enhance the stability of the pulse trains as evident from RF spectra. Simultaneous lowering of timing jitter of the emitted pulses is observed together with a locking in specific ranges of laser injection currents.

Further intensive studies on the beating stability of monolithic mode-locked quantum dot lasers are necessary to understand of the physical processes behind the complex interplay between laser modes and the modes of the auxiliary cavity. Promising lines of investigation include the detailed length dependence of the timing jitter reduction and of the interaction threshold of the pulses propagating inside the laser cavity and the pulses in the auxiliary cavity. Furthermore, it would be useful to modify the feedback arrangement to allow higher feedback levels and the incorporation of an semiconductor optical amplifier.

\section{ACKNOWLEDGMENT}

This work originated from COST Action 288 "Nanoscale and Ultra fast Photonics" via a short term scientific mission (STSM). We acknowledge support by the Optoelectronic Devices group of the COBRA Research Institute at the Technische Universiteit Eindhoven where measurements have been performed.

\section{REFERENCES}

[1] E. V. Andreeva, N. I. Koroteev, S. A. Magnitski, E. Salik, D. S. Starodubov, J. Feinberg, M. V. Shramenko, and S. D. Yakubovich, "Passive mode locking in a multisegment laser diode with an external cavity," Quantum Electron., vol. 29, no. 2, pp. 103-108, 1999.

[2] C. Liu, H. Cao, G. A. Smolyakov, P. G. Eliseev, and M. Osinski, "Anomalous splitting in microwave mode-beating spectra of semiconductor ring lasers," Electron. Lett., vol. 41, no. 17, pp. 963-964, 2005.

[3] R. J. Helkey, D. J. Derickson, A. Mar, J. G. Wasserbauer, J. E. Bowers, and R. L. Thornton, "Repetition frequency stabilization of passively mode-locked semiconductor lasers," Electron. Lett., vol. 28, no. 20, pp. 1920-1922, 1992.

[4] B. Dahmani, L. Hollberg, and R. Drullinger, "Frequency stabilization of semiconductor lasers by resonant optical feedback," Opt. Lett., vol. 12, no. 11, pp. 876-878, 1987.

[5] D. R. Walker, D. W. Crust, W. E. Sleat, and W. Sibbett, "Reduction of phase noise in passively mode-locked," IEEE J. Quantum Electron., vol. 28, no. 1, pp. 289-296, Jan. 1992.

[6] D. J. Jones, K. W. Holman, M. Notcutt, J. Ye, J. Chandalia, L. A. Jiang, E. P. Ippen, and H. Yokoyama, "Ultralow-jitter, 1550-nm mode-locked semiconductor laser synchronized to a visible optical frequency standard," Opt. Lett., vol. 28, no. 10, pp. 813-815, 2003.

[7] D. J. Derickson, R. J. Helkey, A. Mar, J. R. Karin, J. E. Bowers, and R. L. Thornton, "Suppression of multiple pulse formation in external-cavity mode-locked semiconductor lasers using intrawaveguide saturable absorbers," IEEE Photon. Technol. Lett., vol. 4, no. 4, pp. 333-335, Apr. 1992.

[8] E. V. Andreeva, V. K. Batovrin, M. E. Lipin, S. A. Magnitski, E. Salik, D. S. Starodubov, J. Feinberg, M. V. Shramenko, and S. D. Y Qakubovich, "Picosecond semiconductor lasers with an external fibre resonator," Quantum Electron., vol. 30, no. 2, pp. 158-160, 2000.

[9] M. Kuznetsov, D. Z. Tsang, J. N. Walpole, Z. L. Liau, and E. P. Ippen, "Multistable mode locking of InGaAsP semiconductor lasers," Appl. Phys. Lett., vol. 51, no. 12, pp. 895-897, 1987.

[10] L. A. Jiang, K. S. Abedin, M. E. Grein, and E. P. Ippen, "Timing jitter reduction in modelocked semiconductor lasers with photon seeding," Appl. Phys. Lett., vol. 80, no. 10, pp. 1707-1709, 2002.

[11] O. Solgaard and K. Y. Lau, "Optical feedback stabilization of the intensity oscillations in ultrahigh-frequency passively modelocked monolithic quantum-well lasers," IEEE Photon. Technol. Letters, vol. 5, no. 11, pp. 1264-1267, Nov. 1993.

[12] R. F. Broom, E. Mohn, C. Risch, and R. Salathé, "Microwave selfmodulation of a diode laser coupled to an external cavity," IEEE J. Quantum Electron., vol. QE-6, no. 6, pp. 328-334, Jun. 1970.

[13] M. T. Todaro, J.-P. Tourrenc, S. P. Hegarty, C. Kelleher, B. Corbett, G. Huyet, and J. G. McInerney, "Simultaneous achievement of narrow pulsewidth and low pulse-to-pulse timing jitter in $1.3 \mu \mathrm{m}$ passively mode-locked quantum-dot lasers," Opt. Lett., vol. 31, no. 21, pp. 3107-3109, 2006. 
[14] F. Kéfélian, S. O’Donoghue, M. T. Todaro, J. G. McInerney, and G. Huyet, "RF linewidth in monolithic passively mode-locked semiconductor laser," IEEE Photon. Technol. Lett., vol. 20, no. 16, pp. 1405-1407, Nov. 2008.

[15] F. Kéfélian, S. O’Donoghue, M. T. Todaro, J. G. McInerney, and G. Huyet, "Experimental investigation of different regimes of mode-locking in a high repetition rate passively mode-locked semiconductor quantum-dot laser," Opt. Exp., vol. 17, no. 8, pp. 6269-6267, 2009.

[16] C. Liu, P. G. Eliseev, G. A. Smolyakov, H. Cao, and M. Osinski, "Longitudinal mode beating spectra in long-cavity quantum-dot semiconductor lasers," in Proc. Conf. Lasers Electro-Optics/Quantum Electron. Laser Sci. Photon. Appl. Syst. Technol., 2006, Paper JWB2.

[17] P. Schanne, H.-J. Heinrich, W. Elsässer, and E. O. Gobel, "Optical bistability and nearly degenerate four-wave mixing in a GaAlAs laser under intermodal injection," Appl. Phys. Lett., vol. 61, no. 18, pp. 2135-2137, 1992.

[18] H. A. Haus and A. Mecozzi, "Noise of mode-locked lasers," IEEE J. Quantum Electron., vol. 29, no. 3, pp. 983-996, Mar. 1993.

[19] D. Von der Linde, "Characterization of noise in continuously operating mode-locked lasers," Appl. Phys. B, vol. 39, pp. 201-217, 1986.

[20] D. Lenstra, G. Vemuri, and M. Yousefi, , D. M. Kane and K. A. Shore, Eds., "Generalized Optical feedback: Theory," in Unlocking Dynamical Diversity. New York: Wiley, 2005, pp. 58-62.

Stefan Breuer (M'09) received the Dipl. degree in physics from Technical University of Clausthal, Clausthal-Zellerfeld, Germany, in 2005. He is working toward the Ph.D. degree in semiconductor laser optics at Technical University of Darmstadt, Darmstadt, Germany.

Wolfgang Elsäßer (M'94-SM'98) received the Dipl. degree from University of Karlsruhe, Karlsruhe, Germany, in 1980 and the Ph.D. degree from University of Stuttgart, Stuttgart, Germany, in 1984, both in physics.

$\mathrm{He}$ is a Professor in the Institute for Applied Physics, Darmstadt University of Technology, Darmstadt, Germany, heading the Semiconductor Optics Group.

John McInerney received the B.Sc. degree from University College, Cork, Ireland, and the Ph.D. degree from Trinity College, Dublin, Ireland, both in physics.

He has been a Research Engineer at Standard Telecommunication Laboratories, Harlow, U.K., a Research Fellow and Tutor and Fellow of Robinson College, University of Cambridge, U.K., an Associate Professor of electrical engineering and physics at the University of New Mexico, and Director of research at Novalux, Sunnyvale, CA. He is currently Professor and Head of Physics at University College, Cork, Ireland, and also leads the optoelectronics research group at that university's Tyndall Institute. He has over 200 publications and patents in semiconductor laser dynamics and

Dr. McInerney is a Fellow of the Institute of Physics.

Kresten Yvind received the Ph.D. degree from the Technical University of Denmark (DTU), Lyngby, Denmark, in 2003 on a dissertation on semiconductor mode-locked lasers.

$\mathrm{He}$ is currently an Associate Professor at DTU and has broad research interest within semiconductor optoelectronic devices, materials and fabrication technologies.
Jose Pozo was born in Barakaldo, Spain, in 1978. He received the B.Eng. degree from Universidad Publica de Navarra, Pamplona, Spain, and the M.Sc.degree in 2002 from Vrije Universiteit Brussel (Brussels, Belgium). Subsequently, he received the Ph.D. degree from the University of Bristol, U.K., where he studied the temperature dependence of GaInNAs-GaAs quantum-well lasers and developed post-processing techniques using focused ion beam.

In August 2007 he joined the COBRA Research Institute, Eindhoven, The Netherlands, to continue his research in the framework of the projects STWTWICE and STW-EFFECT.

Erwin A. J. M. Bente (M'01) received the Ph.D. degree from the Vrije Universiteit, Amsterdam, The Netherlands, in 1989, for work in atomic laser spectroscopy.

Currently, he is an Associate Professor at the Eindhoven University of Technology, Eindhoven, The Netherlands since 2001. He is working on integrated Indium-phosphide based tunable and short pulse laser systems. From 1988 to 2001, he has worked in industry on laser isotope separation and at the Vrije Universiteit and the Institute of Photonics, University of Strathclyde, Glasgow, U.K., on solid state laser development and non-linear optics.

Mirvais Yousefi (S'00-M'03) received the M.Sc. degree from the University of Lund, Lund, Sweden, in 1998, and the Ph.D. degree from the Vrije Universiteit, Amsterdam, The Netherlands, in 2003.

He worked with the Opto-Electronic Device Group, Technical University of Eindhoven, Eindhoven, The Netherlands, and is currently with TNO-Science and Industry Delft, The Netherlands. His research interests include semiconductor lasers, their dynamics, and the application of these dynamics.

Dr. Yousefi received the IEEE Lasers and Electro-Optics Society Graduate Student Fellowship in 2001.

Asier Villafranca received the M.Sc. and Ph.D. degrees in telecom engineering from the University of Zaragoza, Zaragoza, Spain, in 2004 and 2009, respectively.

Since 2005, he has been with the Photonics Technologies Group, and is currently a Senior Engineer at the Optical Transmission and Broadband Technologies (TOYBA) Laboratory. His research interests include high-resolution and complex optical spectroscopy, semiconductor dynamics and optical network subsystems.

Nikolas Vogiatzis received the Dipl. Ing. in applied maths and physics from National Technical University of Athens, Greece, in 2004. He is currently working toward the Ph.D. on dilute nitride materials at the University of Bristol, Bristol, U.K.

His research interests include optical and transport properties in III-N-V semiconductor materials.

Judy Rorison (M'08) received the D.Phil. in the Theoretical Physics Department at Oxford Univeristy, Oxford, U.K.

She then worked at RSRS, Malvern, followed by Sharp Labs, Oxford, and is currently at the Department of Electrical and Electronic Engineering, University of Bristol, Bristol, U,K. She works on the physics, material science and device design of semiconductor structures for optoelectronic applications. She was the chair of the COST 288 action, Nanoscale and Ultrafast Photonics.

Dr. Rorison is a Fellow of the Institute of Physics. 